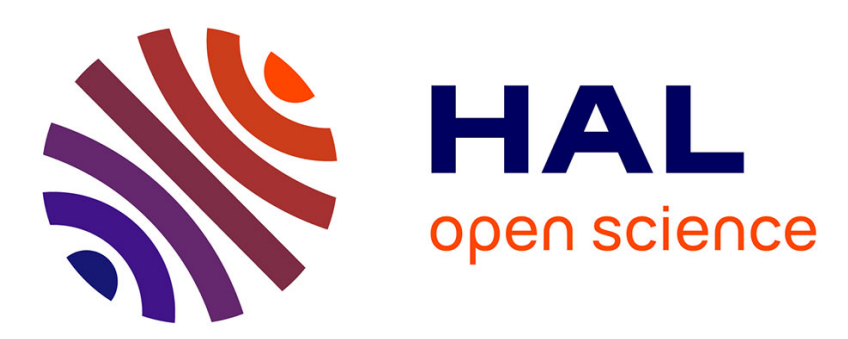

\title{
Double-constrained RPCA based on saliency maps for foreground detection in automated maritime surveillance
}

Andrews Sobral, Thierry Bouwmans, El-Hadi Zahzah

\section{To cite this version:}

Andrews Sobral, Thierry Bouwmans, El-Hadi Zahzah. Double-constrained RPCA based on saliency maps for foreground detection in automated maritime surveillance. ISBC 2015 Workshop conjunction with AVSS 2015, Aug 2015, Karlsruhe, Germany. pp.1-6, 10.1109/AVSS.2015.7301753 . hal01227956

\section{HAL Id: hal-01227956 \\ https://hal.science/hal-01227956}

Submitted on 12 Nov 2015

HAL is a multi-disciplinary open access archive for the deposit and dissemination of scientific research documents, whether they are published or not. The documents may come from teaching and research institutions in France or abroad, or from public or private research centers.
L'archive ouverte pluridisciplinaire HAL, est destinée au dépôt et à la diffusion de documents scientifiques de niveau recherche, publiés ou non, émanant des établissements d'enseignement et de recherche français ou étrangers, des laboratoires publics ou privés. 


\section{Double-constrained RPCA based on Saliency Maps for Foreground Detection in Automated Maritime Surveillance}

\author{
Andrews Sobral \\ Lab. MIA/L3i, Univ. de La Rochelle, France \\ andrews. sobralduniv-lr.fr \\ El-hadi ZahZah \\ Lab. L3i, Univ. de La Rochelle, France \\ ezahzah@univ-lr.fr \\ Thierry Bouwmans \\ Lab. MIA, Univ. de La Rochelle, France \\ tbouwmaneuniv-lr.fr
}

\begin{abstract}
The development of automated video-surveillance applications for maritime environment is a very difficult task due to the complexity of the scenes: moving water, waves, etc. The motion of the objects of interest (i.e. ships or boats) can be mixed with the dynamic behavior of the background (non-regular patterns). In this paper, a double-constrained Robust Principal Component Analysis (RPCA), named SCM-RPCA (Shape and Confidence Mapbased RPCA), is proposed to improve the object foreground detection in maritime scenes. The sparse component is constrained by shape and confidence maps both extracted from spatial saliency maps. The experimental results in the UCSD and MarDT data sets indicate a better enhancement of the object foreground mask when compared with some related RPCA methods.
\end{abstract}

\section{Introduction}

Background subtraction (BS) or foreground separation is commonly the first step in many video surveillance systems to detect moving objects. This basic operation consists of separating the moving objects called "foreground" from the static information called "background". However, in most cases, the background model is not always static due to the complexity of natural scenes: wind in the trees, moving water, waves, etc. A specific case is the development of automated video-surveillance applications for maritime environment (i.e. automatic detection of ships or boats to improve the sea border control).

The maritime surveillance represents a challenging scenario due to the different background dynamics of the observed scenes. Many algorithms have been designed to perform foreground detection $[5,6,13]$, but only a few of them have been designed for maritime scenes. Some related works can be found in Bloisi et al. [3]. The authors propose a multimodal approach for BS to deal with the water background. In addition, Liu et al. [10] proposes an iterative approach for ship target segmentation in infrared images based on multiple features. However, the recent research on subspace estimation by sparse representation and rank minimization show an interesting framework to separate moving objects from the background in videos. The background sequence is modeled by the low-rank subspace that can gradually change over time, while the moving foreground objects constitute the correlated sparse outliers.

Robust Principal Component Analysis (RPCA) solved via Principal Component Pursuit (PCP) [7] decomposes a data matrix $A$ in two components such that $A=L+S$, where $L$ is a low-rank matrix and $S$ is constrained to be a sparse matrix. The low-rank minimization concerning $L$ offers a suitable framework for background modeling due to the correlation between frames. So, minimizing $L$ and $S$ implies that the background is approximated by a low-rank subspace that can gradually change over time, while the moving foreground objects constitute the correlated sparse outliers which are contained in $S$. To obtain the foreground mask, $S$ needs to be thresholded. Usually, the threshold is determined experimentally.

However, PCP is limited to the low-rank component being exactly low-rank and the sparse component being exactly sparse but the observations in real applications are often corrupted by noise affecting every entry of the data matrix. Therefore, Zhou et al. [18] proposed a stable PCP (SPCP) that guarantee stable and accurate recovery in the presence of entry-wise noise. SPCP assumes that the observation matrix $A$ is represented as $A=L+S+E$ (also named as three-term decomposition), where $E$ is a noise term. To recover $L, S$ and $E$, Zhou et al. [18] proposed to solve the following optimization problem, as a relaxed ver- 


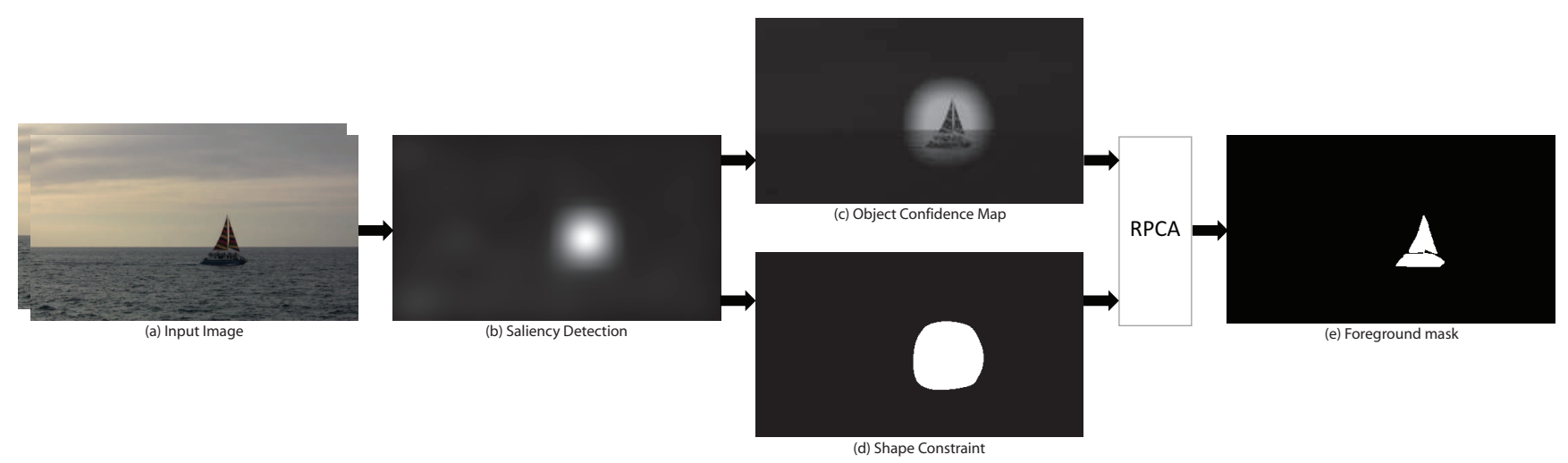

Figure 1. Block diagram of the proposed approach. Given an input image (a), a saliency detector is applied (b). Next, the confidence map (c) is built by normalizing the saliency map, while the shape constraint (or region of interest) (d) is built by thresholding this one, and (e) the foreground mask obtained by thresholding the RPCA sparse component. Note that in some environments where a) the object of interest move very slowly (i.e long distance boats), or b) the background is very dynamic (i.e boats in the sea), the optical flow may not be enough to ensure the object detection. In addition, the double constraints (confidence map and shape) can be built from two different types of source (i.e. from spatial, temporal, or spatio-temporal information), but in this work we focus only on spatial saliency maps.

sion to PCP: $\min _{L, S, E}\|L\|_{*}+\lambda\|S\|_{1}+\gamma\|E\|_{F}^{2}$, s.t. $A=L+$ $S+E$, where $\|\cdot\|_{*},\|\cdot\|_{1}$ and $\|\cdot\|_{F}$ are the nuclear norm (i.e. sum of singular values), $l_{1}$-norm (sum of matrix elements magnitude) and the Frobenius norm, respectively, while $\lambda>0$ and $\gamma>0$ are an arbitrary weighting parameter. This decomposition is called "stable" decomposition as it separates the outliers in $S$ and the noise in $E$.

In scenes where the background is very dynamic (i.e. sea waves in maritime surveillance [3]), the motion of the objects of interest (i.e. boats) will be mixed with the dynamic behavior of the background (i.e. waves). SPCP-based methods tries to deal with this problem under the term where the multi-modality of the background (i.e. waves) is considered as noise component $(E)$, while the moving objects (i.e. boats) is considered as sparse component $(S)$. The low-rank component $(L)$ represents the static part of the background.

In this paper, a double-constrained RPCA, named SCMRPCA (Shape and Confidence Map-based RPCA), is proposed to improve the object foreground detection in dynamic scenes. The sparse component is constrained by shape and confidence maps both extracted from spatial saliency maps. One advantage of the SCM-RPCA in relation to its direct competitors, is the possibility to combine two different types of source: spatial, temporal, and spatiotemporal information, but in this work we focus only on spatial saliency maps. Fig. 1 highlights our proposed approach. Given an input image (a), a saliency detector is applied (b). Next, the confidence map (c) is built by normalizing the saliency map, while the shape constraint (d) is built by thresholding this one, and (e) the foreground mask given by thresholding the RPCA sparse component.

First, we start with the related works (Section 2), and next we describe our proposed approach (Section 3). The remainder of the paper is organized as follows: Section 4 describes how to solve our SCM-RPCA algorithm. Section 5 defines the concept of shape and confidence map. Finally, in Sections 6 and 7, the experimental results are shown as well as conclusions.

\section{Related works}

In the literature, there are several modifications which concern the original SPCP. Some authors $[12,14,15]$ added constraint in the sparse term in order to improve the foreground detection. First, Oreifej et al. [12] use a turbulence model to enforce an additional constraint on the rank minimization. The authors quantify the scenes motion in terms of the motion of the particles which are driven by dense optical flow. The obtained confidence map (a real-valued matrix) provides a rough prior knowledge of the moving objects locations, which can be incorporated into the matrix optimization problem. Subsequently, Yang et al. $[14,15]$ propose a motion-assisted matrix restoration (MAMR) model for foreground-background separation. Thus, a dense motion field is estimated for each frame by dense optical flow, and mapped into a weighting matrix which indicates the likelihood that each pixel belongs to the background. By incorporating this information, areas dominated by slowly-moving objects are suppressed while the background that appears at only a few frames has more chances to be recovered in the foreground detection results. In addition, Yang et al. [15] extended MAMR (RMAMR) which is robust approach to noise for practical applications.

\section{SCM-RPCA approach}

In this work, we propose to combine some ideas proposed by both Oreifej et al. [12] and Yang et al. [14, 15]. The weighting matrix proposed by Yang et al. [14, 15] 


\begin{tabular}{ll}
\hline Author(s) & Minimization \\
\hline Oreifej et al. [12] & $\min _{L, S, E}\|L\|_{*}+\lambda\|\Pi(S)\|_{1}+\gamma\|E\|_{F}^{2}$ \\
& s.t. $A=L+S+E$ \\
Yang et al. [15] & $\min _{L, S, E}\|L\|_{*}+\lambda\|S\|_{1}+\gamma\|E\|_{F}^{2}$ \\
& s.t. $W \circ A=W \circ(L+S+E)$ \\
SCM-RPCA & $\begin{array}{l}\min _{L, S, E}\|L\|_{*}+\lambda\|\Pi(S)\|\left\|_{1}+\gamma\right\| E \|_{F}^{2} \\
\text { s.t. } A=L+W \circ S+E\end{array}$ \\
\hline Table 1. Comparison of the proposed method and related works.
\end{tabular}

can be used as a shape constraint (or region constraint), while the confidence map proposed by Oreifej et al.[12] reinforces the pixels belonging from the moving objects. A modified version of the original $3 \mathrm{WD}$ method was implemented adding the shape constraint as has been done in the RMAMR. Part of the reason we chose to modify the $3 \mathrm{WD}$ instead of RMAMR is it capacity to deal with the multimodality of the background. The second contribution of this paper refers to the way of building the shape constraint and confidence map. Instead of using dense optical flow (temporal descriptor) as a preliminary step, we suggest to use a saliency detector (spatial descriptor). In some cases where a) the object of interest moves very slowly (i.e long distance boats) or b) the background is very dynamic (i.e boats in the sea), the optical flow may not be enough to ensure the object detection. In addition, computing the dense optical flow request high computational cost, while computing the saliency map is commonly much more fast. Several saliency detection methods have been proposed in the literature [4]. In this work, the BMS ${ }^{1}$ method proposed by Zhang and Sclaroff $[16,17]$ was selected by its speed performance and visual results.

Let a sequence of $k$ gray-scale images (frames) $I_{1} \ldots I_{k}$ captured from a static camera, that is, $I \in \mathbb{R}^{m \times n}$ where $m$ and $n$ denotes the frame resolution (rows by columns). All frames are vectorized into a observation matrix $A=$ $\left[\operatorname{vec}\left(I_{1}\right) \ldots \operatorname{vec}\left(I_{k}\right)\right]$, where $A \in \mathbb{R}^{m n \times k}$ and $m n=(m \times$ $n)$. Our decomposition can be formulated as:

$$
\begin{aligned}
& \min _{L, S, E}\|L\|_{*}+\lambda\|\Pi(S)\|_{1}+\gamma\|E\|_{F}^{2}, \\
& \text { s.t. } A=L+W \circ S+E
\end{aligned}
$$

where $\Pi \in \mathbb{R}^{m n \times k}$ and $W \in[0,1]^{m n \times k}$ are the confidence map and shape constraint (binary map), respectively, and "o" denotes element-wise multiplication of two matrix. As explained previously, the confidence map $\Pi$ reinforce the pixels belonging from the moving objects and the shape constraint $W$ defines the region of interest. Table 1 shows

\footnotetext{
${ }^{1}$ http://cs-people.bu .edu/jmzhang/BMS/BMS . html
}

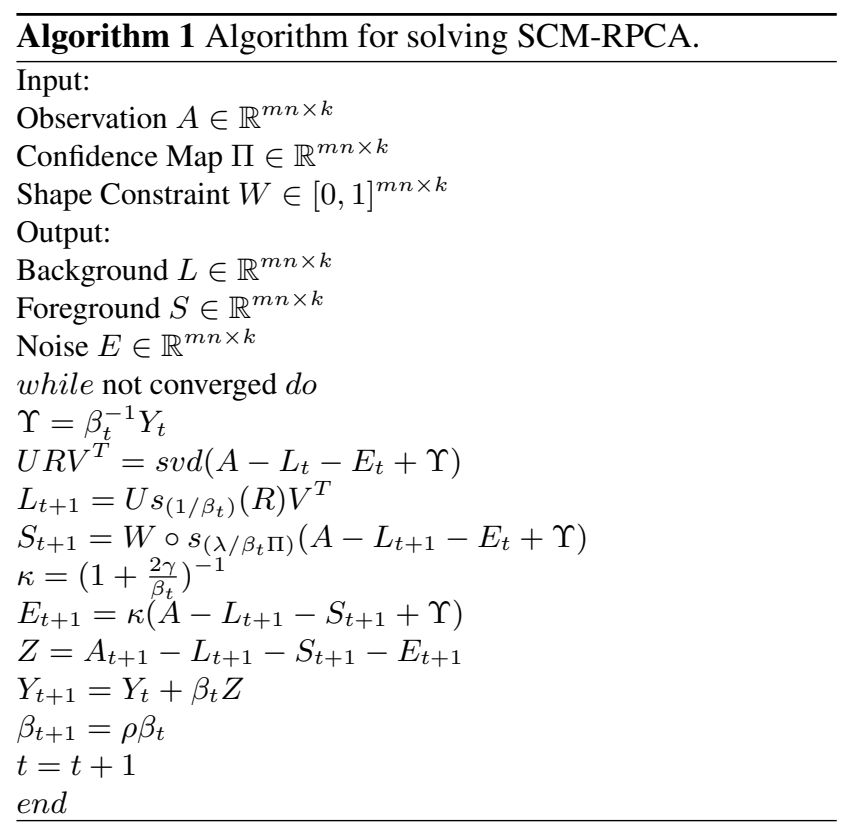

the comparison of the proposed method between Oreifej et al. [12] and Yang et al. [14, 15]. These minimization problems are convex and can be solved by the Alternating Direction Method (ADM) under the Augmented Lagrangian Multiplier (ALM) framework [9].

\section{Solving the SCM-RPCA}

To solve the Equation 1, the ALM [9] is used. The ALM framework convert the constrained optimization problem to the minimization of the augmented Lagrange function:

$$
\begin{aligned}
& \mathcal{L}(L, S, E, Y)=\|L\|_{*}+\lambda\|\Pi(S)\|_{1}+\gamma\|E\|_{F}^{2} \\
& +<Y, A-L-W \circ S-E)> \\
& +\frac{\beta}{2}\|(A-L-W \circ S-E)\|_{F}^{2}
\end{aligned}
$$

where $Y \in \mathbb{R}^{m n \times k}$ is a Lagrange multiplier matrix, $\beta>0$ is the penalty parameter for the violation of the linear constraint, and $<,>$ denotes the matrix inner product. Next, the ADM is used to solve $L, S, E$ and $Y$ alternatingly:

$$
\begin{aligned}
& L_{t+1}=\underset{L}{\operatorname{argmin}} \mathcal{L}\left(L, S_{t}, E_{t}, Y_{t}\right), \\
& S_{t+1}=\underset{S}{\operatorname{argmin}} \mathcal{L}\left(L_{t+1}, S, E_{t}, Y_{t}\right), \\
& E_{t+1}=\underset{E}{\operatorname{argmin}} \mathcal{L}\left(L_{t+1}, S_{t+1}, E, Y_{t}\right), \\
& Z=\left(A_{t+1}-L_{t+1}-S_{t+1}-E_{t+1}\right), \\
& Y_{t+1}=Y_{t}+\beta_{t} Z
\end{aligned}
$$

where $Z \in \mathbb{R}^{m n \times k}$ is the residuals. Then, a closed form solution for each of minimization problems can be defined by: 


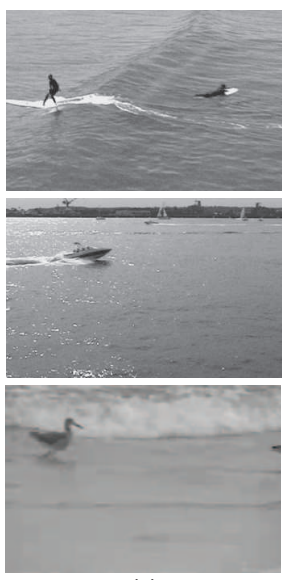

(a)
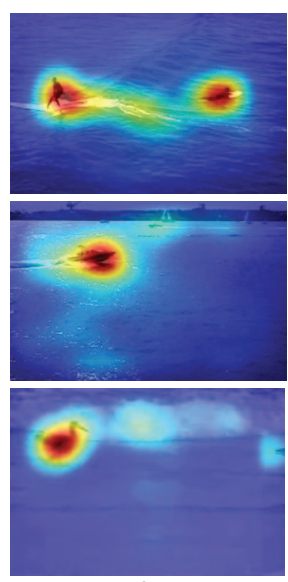

(b)

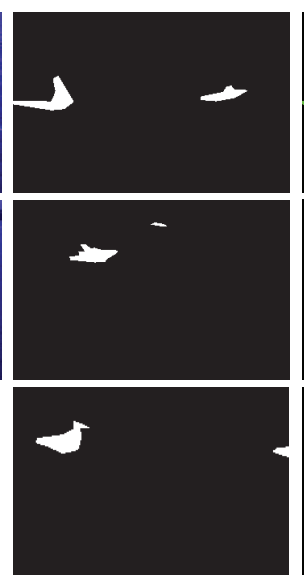

(c)

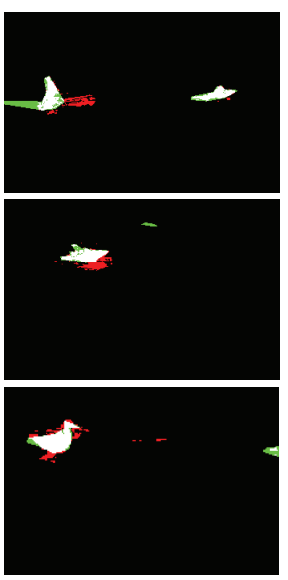

(d)

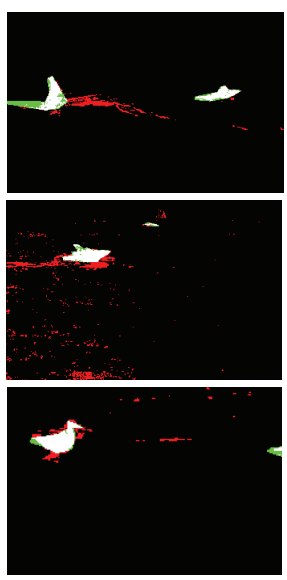

(e)

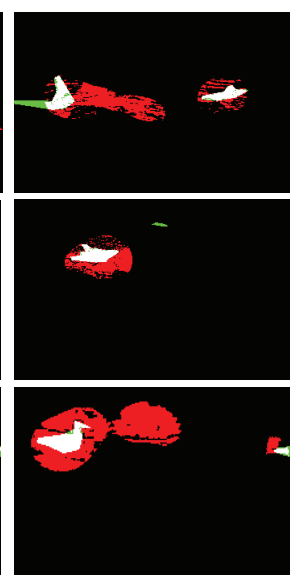

(f)

Figure 2. Visual comparison on background subtraction results over three scenes of UCSD data set. From top to bottom: surfers, boats and birds. From left to right: (a) input frame, (b) saliency map generated by BMS [17], (c) ground truth, (d) proposed approach, (e) 3WD [12], and (f) RMAMR [15]. The top 3 best algorithms (organized by rank) from Table 2 was chosen. The true positives (TP) pixels are in white, true negatives $(T N)$ pixels in black, false positives $(F P)$ pixels in red and false negatives $(F N)$ pixels in green.

$$
\begin{aligned}
& \Upsilon=\beta_{t}^{-1} Y_{t} \\
& U R V^{T}=\operatorname{svd}\left(A-L_{t}-E_{t}+\Upsilon\right), \\
& L_{t+1}=U s_{\left(1 / \beta_{t}\right)}(R) V^{T} \\
& S_{t+1}=W \circ s_{\left(\lambda / \beta_{t} \Pi\right)}\left(A-L_{t+1}-E_{t}+\Upsilon\right), \\
& \kappa=\left(1+\frac{2 \gamma}{\beta_{t}}\right)^{-1} \\
& E_{t+1}=\kappa\left(A-L_{t+1}-S_{t+1}+\Upsilon\right)
\end{aligned}
$$

where $\operatorname{svd}($.$) denotes a full singular value decomposition,$ and $s_{(.)}($.$) is the soft thresholding operator defined by:$

$$
s_{(\alpha)}(X)=\operatorname{sign}(X) \max (\operatorname{abs}(X)-\alpha, 0)
$$

where $s_{(.)}($.$) is applied in a matrix X$ in an element-wise manner. The main steps of our proposed algorithm are shown in Algorithm 1. Usually the convergence is done when $\left(\|Z\|_{F} /\|A\|_{F}\right)<\epsilon$ (where $\epsilon$ is the error tolerance), or when the \# of iterations is reached. The parameters $\lambda$ and $\gamma$ are scalars and defines the weighting parameter for the sparse and noise component, respectively, and $\rho$ is a constant scalar and growth factor for the $\beta$ parameter. Oreifej et al. [12] shows when $\beta_{t}$ is a monotonically increasing positive sequence, the iterations converge to the optimal solution of problem 1 . In this work, $\lambda, \gamma, \rho$, and $\beta_{0}$ were defined empirically as $2,1 /\|A\|_{2}, 1.25$, and $5 / \sqrt{m}$, respectively.

\section{Definition of shape and confidence map}

In this work, both the confidence map $\Pi$ and the shape constraint $W$ are constructed from spatial information given by saliency maps instead of optical flow, as proposed originally by Oreifej et al. [12] and Yang et al. [14, 15]. Let a sequence of $k$ saliency maps denoted by $M_{1} \ldots M_{k}$ where $M \in \mathbb{R}^{m \times n}$, so:

$$
\begin{aligned}
\Pi & =\left[\operatorname{vec}\left(\operatorname{norm}\left(M_{1}\right)\right) \ldots \operatorname{vec}\left(\operatorname{norm}\left(M_{k}\right)\right)\right] \\
W & =\left[\operatorname{vec}\left(\operatorname{thresh}\left(M_{1}\right)\right) \ldots \operatorname{vec}\left(\operatorname{thresh}\left(M_{k}\right)\right)\right]
\end{aligned}
$$

where norm (.) denotes the min-max normalization, scaling all entries of $M$ between 0 and 1 by:

$$
\operatorname{norm}\left(M_{(i, j)}\right)=\frac{M_{(i, j)}-M_{m i n}}{M_{m a x}-M_{m i n}}
$$

with $M_{\min }$ and $M_{\max }$ the minimum value and the maximum value of the matrix $M$, respectively, and $M_{(i, j)}$ the matrix element located in the row $i \in[1 \ldots m]$ and column $j \in[1 \ldots n]$. Subsequently, thresh(.) denotes the thresholding function defined as:

$$
\operatorname{thresh}\left(M_{(i, j)}\right)= \begin{cases}1 & \text { if }\left(0.5 M_{(i, j)}\right)^{2}<\mu \\ 0 & \text { otherwise }\end{cases}
$$

where $\mu=0.5 \eta(\operatorname{std}(\operatorname{vec}(M)))^{2}$, and $\operatorname{std}($.$) denotes the$ standard deviation of a data vector. In this work, $\eta$ was chosen experimentally and defined as 10 .

\section{Experimental results}

In order to evaluate the performance of the proposed method for background subtraction, four videos extracted from the UCSD Background Subtraction Dataset ${ }^{2}$ proposed by Mahadevan and Vasconcelos [11] and three videos from MarDT data $\operatorname{set}^{3}$ proposed by Bloisi et al. [2] was selected.

\footnotetext{
${ }^{2}$ http: //www.svcl.ucsd.edu/projects/background _subtraction/ucsdbgsub\_dataset.htm

${ }^{3}$ http: //www.dis.uniroma1.it/ labrococo/MAR/ index.htm
} 
Table 2. Quantitative results on four videos of UCSD Background Subtraction Dataset [11].

\begin{tabular}{|c|c|c|c|c|c|c|c|c|c|c|c|c|c|}
\hline & \multicolumn{3}{|c|}{ Birds } & \multicolumn{3}{|c|}{ Surfers } & \multicolumn{3}{|c|}{ Boats } & \multicolumn{3}{|c|}{ Ocean } & Rank \\
\hline & $R e$ & $\operatorname{Pr}$ & $F_{1}$ & $R e$ & $\operatorname{Pr}$ & $F_{1}$ & $R e$ & $\mathrm{Pr}$ & $F_{1}$ & $R e$ & $\operatorname{Pr}$ & $F_{1}$ & $A v g . F_{1}$ \\
\hline PCP [7] & 0.842 & 0.094 & 0.170 & 0.754 & 0.075 & 0.137 & 0.814 & 0.100 & 0.178 & 0.748 & 0.115 & 0.200 & 0.171 \\
\hline Lag-SPCP-QN [1] & 0.413 & 0.322 & 0.362 & 0.244 & 0.282 & 0.261 & 0.405 & 0.215 & 0.281 & 0.484 & 0.313 & 0.380 & 0.321 \\
\hline RMAMR [15] & 0.823 & 0.229 & 0.358 & 0.775 & 0.248 & 0.376 & 0.816 & 0.230 & 0.359 & 0.777 & 0.175 & 0.286 & 0.345 \\
\hline $3 \mathrm{WD}[12]$ & 0.586 & 0.604 & 0.595 & 0.538 & 0.405 & 0.462 & 0.673 & 0.473 & 0.556 & 0.563 & 0.337 & 0.422 & 0.509 \\
\hline SCM-RPCA & 0.573 & 0.638 & 0.604 & 0.518 & 0.565 & 0.541 & 0.663 & 0.550 & 0.602 & 0.457 & 0.544 & 0.497 & 0.561 \\
\hline
\end{tabular}

Table 3. Benchmark evaluation over four videos of UCSD Background Subtraction Dataset [11].

\begin{tabular}{lrrrrrrrr}
\hline & \multicolumn{2}{c}{ Birds } & \multicolumn{2}{c}{ Surfers } & \multicolumn{2}{c}{ Boats } & \multicolumn{2}{c}{ Ocean } \\
& $(242 \times 156 \times 71)$ & $(344 \times 224 \times 41)$ & $(344 \times 224 \times 31)$ & $(316 \times 196 \times 176)$ \\
\hline & Iter & Time $^{*}$ & Iter & Time $^{*}$ & Iter & Time $^{*}$ & Iter $^{\text {Time }^{*}}$ \\
PCP [7] & +100 & 27.29 & +100 & 21.19 & +100 & 18.47 & +100 & 110.53 \\
Lag-SPCP-QN [1] & $\mathbf{2 9}$ & 10.12 & 53 & 16.27 & 39 & 10.01 & $\mathbf{1 8}$ & $\mathbf{2 9 . 4 9}$ \\
RMAMR [15] & 34 & 10.63 & 35 & 13.09 & 33 & 11.44 & 35 & 44.22 \\
3WD [12] & 30 & $\mathbf{4 . 5 3}$ & 26 & $\mathbf{4 . 2 8}$ & 31 & 4.06 & 42 & 29.96 \\
SCM-RPCA & $\mathbf{2 9}$ & 4.59 & $\mathbf{2 5}$ & 4.37 & $\mathbf{2 7}$ & $\mathbf{3 . 8 2}$ & 43 & 33.02 \\
\hline
\end{tabular}

$($ width $\times$ height $\times$ length $)$ denotes the frame resolution and the number of processed frames.

* Time for matrix decomposition (in seconds). Does not include the time to compute the input constraint (saliency maps).

+ Iteration limit 100 reached.

Table 4. Precision, Recall and F-Measure metrics.

\begin{tabular}{ll}
\hline Metrics & Description \\
\hline Precision $(P r)$ & $T P /(T P+F P)$ \\
Recall $(R e)$ & $T P /(T P+F N)$ \\
F-Measure $\left(F_{1}\right)$ & $2 \times(P r \times R e) /(P r+R e)$ \\
\hline$T P=$ \# of foreground pixels classified as foreground. \\
$F P=$ \# of background pixels classified as foreground. \\
$T N=$ \# of background pixels classified as background. \\
$F N=$ \# of foreground pixels classified as background.
\end{tabular}

The UCSD and MarDT data sets consists of 18 and 28 video sequences, respectively, both acquired from a stationary and moving camera, but in this work (due to page limits) we have selected only the four sequences from UCSD and three sequences from MarDT.

We have compared the SCM-RPCA with its direct competitors: the orignal PCP proposed by Candès et al. [7], the stable PCP proposed by Aravkin et al. [1], the 3WD proposed by Oreifej et al. [12], and the RMAMR proposed by Yang et al. [15]. Note that the PCP and stable PCP are not constrained, while 3WD and RMAMR are singleconstrained RPCA. Is important to note that all constrained RPCA evaluated in this paper have been used the saliency maps as the input constraint.

In the next sections, we report the quality evaluation and the benchmark evaluation of the selected algorithms.

\subsection{Quality evaluation}

Figures 2 and 3 show the visual results for background subtraction task in the UCSD and MarDT data sets, respec- tively. Is important to note that in the UCSD scenes we have used the original spatial saliency map provided by BMS, while for the MarDT scenes we have subtracted its temporal median due to the high saliency from the buildings around the river. The quantitative results in Table 2 show that the SCM-RPCA outperforms the previous methods, with the highest $F$-measure average over the selected video sequences. Each metric is described in Table 4. As can be seen from Figures 2 and 3, and Table 2, the combination with confidence map and shape constraint can reduce the amount of false positive pixels.

\subsection{Benchmark evaluation}

In Table 3, we report our benchmark evaluation over four videos of UCSD Background Subtraction Dataset [11]. The algorithms are implemented in MATLAB (R2014a) running on a laptop computer with Windows 7 Professional 64 bits, $2.7 \mathrm{GHz}$ Core i7-3740QM processor and 32Gb of RAM. Note that in Table 3 the number of iterations (Iter) of the proposed method is slightly less than the 3WD and RMAMR, except for the Ocean scene. However, the computation time is slightly increased, except for the Boats scene. We noticed that the combination of shape constraint and confidence map did not changed significantly the number of iterations and computation time over original 3WD.

\section{Conclusion}

In this paper a double-constrained version of RPCA is proposed to improve the foreground detection in dynamic scenes. The sparse component is constrained by shape and confidence maps both extracted from spatial saliency maps. 


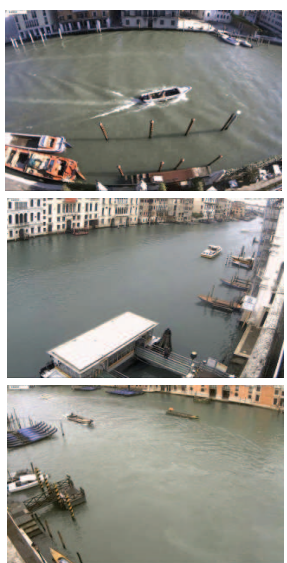

(a)

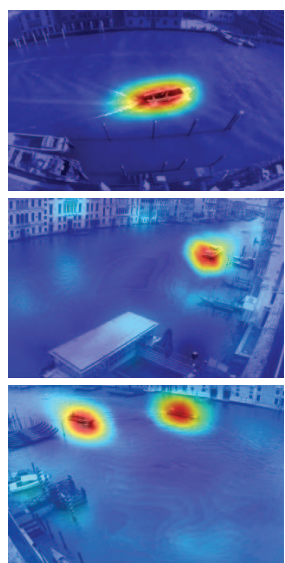

(b)

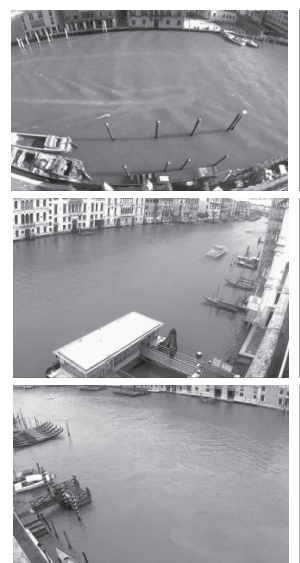

(c)

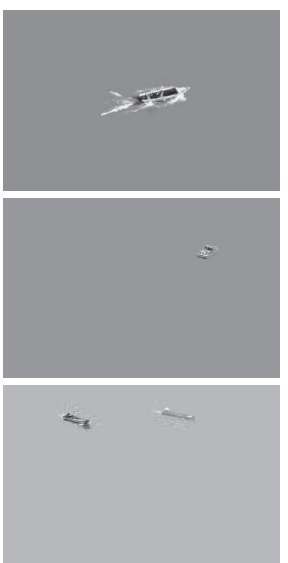

(d)

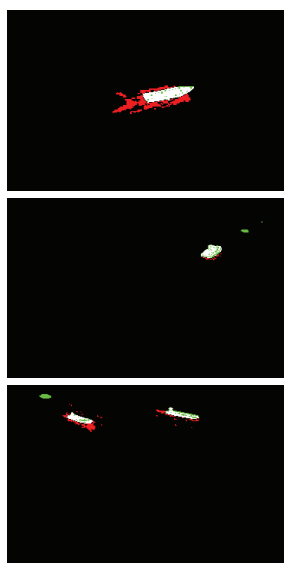

(e)

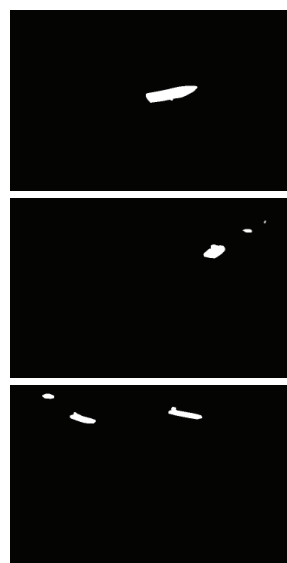

(f)

Figure 3. Visual results of SCM-RPCA over three scenes of MarDT data set. From left to right: (a) input frame, (b) saliency map with its temporal median subtracted (due to the high saliency from the buildings around the river), (c) low-rank component, (d) sparse component, (e) foreground mask, and (f) ground truth. The true positives $(T P)$ pixels are in white, true negatives $(T N)$ pixels in black, false positives $(F P)$ pixels in red and false negatives $(F N)$ pixels in green.

The experimental results indicate a better enhancement of the object foreground mask when compared with its direct competitors. As shown in quality evaluation, the combination with confidence map and shape constraint can reduce the amount of false positive pixels. In addition, our benchmark evaluation demonstrates that the proposed algorithm have a slightly change in the number of iterations and computation time compared to the original 3WD.

In the future work, we will investigate how spatiotemporal saliency detectors can help the proposed approach to improve the foreground detection. In this work, the confidence map and shape constraint were built from the same source, specifically by saliency maps. We will explore how different sources can be used to built separately these constraints. Furthermore, we are interested in adapting the proposed algorithm to perform the decomposition incrementally as in Online RPCA [8]. MATLAB codes available at ${ }^{4}$.

\section{References}

[1] A. Y. Aravkin, S. Becker, V. Cevher, and P. Olsen. A variational approach to stable principal component pursuit. The Conf. on Uncertainty in Artificial Intelligence, 2014.

[2] D. D. Bloisi, L. Iocchi, and A. Pennisi. Mar - maritime activity recognition dataset. http://www. dis.uniromal. it/ bloisi/mar, Jan. 2013.

[3] D. D. Bloisi, A. Pennisi, and L. Iocchi. Background modeling in the maritime domain. Machine Vision and Applications, 25(5):1257-1269, 2014.

[4] A. Borji, M. Cheng, H. Jiang, and J. Li. Salient object detection: A survey. CoRR, abs/1411.5878, 2014.

[5] T. Bouwmans. Traditional and recent approaches in background modeling for foreground detection: An overview. In Computer Science Review, 2014.

\footnotetext{
${ }^{4}$ https://sites.google.com/site/scmrpca
}

[6] T. Bouwmans and E. Zahzah. Robust PCA via Principal Component Pursuit: A review for a comparative evaluation in video surveillance. In CVIU, May 2014.

[7] E. Candes, X. Li, Y. Ma, and J. Wright. Robust principal component analysis? Int. Journal of ACM, 58(3):117-142, May 2011.

[8] J. Feng, H. Xu, and S. Yan. Online robust pca via stochastic optimization. In NIPS, pages 404-412, 2013.

[9] Z. Lin, M. Chen, and Y. Ma. The Augmented Lagrange Multiplier Method for Exact Recovery of Corrupted Low-Rank Matrices. Mathematical Programming, 2010.

[10] Z. Liu, F. Zhou, X. Chen, X. Bai, and C. Sun. Iterative infrared ship target segmentation based on multiple features. Pattern Recognition, 47(9):2839-2852, 2014.

[11] V. Mahadevan and N. Vasconcelos. Spatiotemporal saliency in dynamic scenes. IEEE PAMI, 32(1):171-177, 2010.

[12] O. Oreifej, X. Li, and M. Shah. Simultaneous video stabilization and moving object detection in turbulence. IEEE PAMI, 35(2):450-462, Feb 2013.

[13] A. Sobral and A. Vacavant. A comprehensive review of background subtraction algorithms evaluated with synthetic and real videos. CVIU, 122(0):4-21, 2014.

[14] J. Yang, X. Sun, X. Ye, and K. Li. Background extraction from video sequences via motion-assisted matrix completion. In IEEE ICIP'2014, pages 2437-2441, Oct 2014.

[15] X. Ye, J. Yang, X. Sun, K. Li, C. Hou, and Y. Wang. Foreground-background separation from video clips via motion-assisted matrix restoration. IEEE Trans. on CSVT, PP(99):1-1, 2015.

[16] J. Zhang and S. Sclaroff. Saliency detection: a boolean map approach. In IEEE ICCV, 2013.

[17] J. Zhang and S. Sclaroff. Exploiting surroundedness for saliency detection: a boolean map approach. In IEEE PAMI, 2014.

[18] Z. Zhou, X. Li, J. Wright, E. J. Candès, and Y. Ma. Stable principal component pursuit. CoRR, abs/1001.2363, 2010. 